Supporting Information for:

\title{
Solution NMR and computer simulation studies of active site loop motion in triosephosphate isomerase.
}

Francesca Massi, Chunyu Wang, and Arthur G. Palmer, III

Table 1: Backbone ${ }^{1} \mathrm{H}$ and ${ }^{15} \mathrm{~N}$ resonance assignments of G3P bound TIM

Table 2: Conformational exchange contribution to transverse relaxation, $R_{e x}$, for G3P bound TIM at $298 \mathrm{~K}, 293 \mathrm{~K}, 288 \mathrm{~K}$, and $283 \mathrm{~K}$ at $B_{0}=18.8 \mathrm{~T}$. 


\begin{tabular}{|c|c|c|c|c|c|c|c|c|c|c|c|}
\hline residue & ${ }^{15} \mathrm{~N}$ & ${ }^{1} \mathrm{H}^{N}$ & residue & ${ }^{15} \mathrm{~N}$ & ${ }^{1} \mathrm{H}^{N}$ & residue & ${ }^{15} \mathrm{~N}$ & ${ }^{1} \mathrm{H}^{N}$ & residue & ${ }^{15} \mathrm{~N}$ & ${ }^{1} \mathrm{H}^{N}$ \\
\hline T4 & 125.1 & 9.11 & $\overline{~ G 72}$ & 105.8 & 7.92 & בA136 & 119.2 & 7.20 & G197 & 110.0 & 7.43 \\
\hline F5 & 131.2 & 8.69 & A73 & 128.6 & 8.08 & G137 & 108.4 & 8.00 & D198 & 120.3 & 8.21 \\
\hline F6 & 130.8 & 7.46 & F74 & 120.7 & 6.59 & K138 & 117.5 & 8.10 & K199 & 122.3 & 8.45 \\
\hline N10 & 123.9 & 9.45 & G76 & 111.7 & 9.39 & $\mathrm{~T} 139$ & 116.1 & 7.56 & A200 & 122.3 & 8.45 \\
\hline K12 & 116.6 & 8.06 & E77 & 120.8 & 7.26 & V142 & 120.6 & 8.23 & A201 & 118.9 & 7.66 \\
\hline L13 & 127.0 & 7.93 & N78 & 115.5 & 9.45 & V143 & 115.2 & 7.95 & S202 & 112.7 & 7.97 \\
\hline N14 & 117.5 & 7.59 & S79 & 115.0 & 8.21 & E144 & 119.9 & 8.21 & E203 & 122.1 & 7.23 \\
\hline G15 & 106.5 & 8.34 & V80 & 126.7 & 9.84 & $\mathrm{R} 145$ & 121.7 & 7.58 & L204 & 123.2 & 6.85 \\
\hline K17 & 124.2 & 10.14 & D81 & 121.1 & 7.67 & Q146 & 119.5 & 8.34 & $\mathrm{R} 205$ & 126.6 & 8.85 \\
\hline Q18 & 118.3 & 8.30 & Q82 & 122.1 & 6.93 & N148 & 121.1 & 9.52 & D213 & 119.8 & 8.88 \\
\hline K21 & 121.6 & 7.78 & K84 & 118.7 & 7.60 & A149 & 120.0 & 7.51 & N213 & 120.4 & 9.39 \\
\hline E22 & 117.1 & 7.14 & D85 & 122.1 & 7.77 & V150 & 117.2 & 6.92 & G214 & 105.2 & 9.73 \\
\hline I 23 & 120.6 & 7.79 & V86 & 106.0 & 7.21 & L151 & 122.1 & 8.75 & $\mathrm{~S} 215$ & 116.2 & 8.38 \\
\hline N28 & 115.8 & 8.55 & G87 & 106.9 & 7.67 & D156 & 118.0 & 7.13 & $\mathrm{~A} 217$ & 129.9 & 8.37 \\
\hline T29 & 109.8 & 7.62 & G94 & 106.5 & 9.21 & $\mathrm{~F} 157$ & 129.6 & 9.38 & V218 & 117.6 & 8.51 \\
\hline A30 & 127.2 & 7.66 & H95 & 119.0 & 7.51 & $\mathrm{~T} 158$ & 120.3 & 8.41 & $\mathrm{~T} 219$ & 113.2 & 7.75 \\
\hline S31 & 116.6 & 8.41 & S100 & 111.3 & 8.26 & N159 & 119.3 & 9.25 & $\mathrm{~F} 220$ & 118.8 & 7.84 \\
\hline I32 & 124.1 & 7.86 & Y101 & 118.6 & 7.74 & V167 & 129.0 & 8.38 & $\mathrm{~K} 221$ & 122.2 & 7.61 \\
\hline E34 & 119.0 & 8.56 & F102 & 111.9 & 6.49 & W168 & 114.1 & 7.01 & A 224 & 128.5 & 8.42 \\
\hline N35 & 116.2 & 8.19 & H103 & 112.5 & 7.06 & A169 & 125.5 & 7.15 & D225 & 114.0 & 9.81 \\
\hline V36 & 116.0 & 6.90 & E104 & 119.7 & 7.67 & $\mathrm{I} 170$ & 121.3 & 7.04 & V226 & 120.9 & 7.47 \\
\hline V38 & 124.1 & 8.15 & D105 & 124.5 & 8.11 & $\mathrm{~T} 172$ & 108.2 & 6.80 & D227 & 125.7 & 8.65 \\
\hline $\mathrm{T} 45$ & 111.2 & 7.50 & D106 & 117.5 & 8.14 & G173 & 112.0 & 8.36 & L230 & 125.5 & 8.34 \\
\hline L47 & 126.6 & 7.40 & K107 & 120.2 & 7.97 & L174 & 125.8 & 7.72 & V231 & 126.6 & 9.00 \\
\hline D48 & 120.3 & 9.05 & F108 & 123.2 & 8.18 & A175 & 122.9 & 7.62 & $\mathrm{~S} 235$ & 115.5 & 8.24 \\
\hline Y49 & 119.6 & 9.08 & A110 & 127.9 & 8.65 & A176 & 123.3 & 8.18 & L236 & 118.4 & 6.55 \\
\hline L53 & 120.9 & 7.18 & D111 & 120.1 & 8.86 & $\mathrm{~T} 177$ & 113.1 & 8.73 & $\mathrm{~K} 237$ & 118.1 & 7.08 \\
\hline V54 & 122.8 & 7.32 & G118 & 108.5 & 7.66 & E179 & 115.8 & 8.12 & E239 & 112.7 & 7.93 \\
\hline K55 & 127.4 & 8.82 & Q119 & 117.3 & 7.16 & D180 & 122.8 & 8.01 & F240 & 125.1 & 7.95 \\
\hline K56 & 122.6 & 6.87 & G120 & 106.7 & 7.58 & A181 & 121.7 & 8.50 & V241 & 114.7 & 7.54 \\
\hline Q58 & 116.2 & 8.83 & V121 & 118.3 & 7.20 & Q182 & 117.1 & 8.04 & D242 & 120.3 & 6.95 \\
\hline T60 & 119.8 & 8.85 & G128 & 108.8 & 9.51 & $\mathrm{I} 184$ & 122.3 & 8.14 & $\mathrm{I} 243$ & 119.5 & 7.45 \\
\hline V61 & 121.3 & 8.72 & E129 & 128.2 & 9.79 & H185 & 122.1 & 9.63 & $\mathrm{I} 244$ & 123.3 & 7.40 \\
\hline Q64 & 117.6 & 9.92 & $\mathrm{~T} 130$ & 113.0 & 9.52 & A186 & 121.5 & 8.70 & N245 & 114.9 & 7.31 \\
\hline A66 & 121.4 & 7.23 & L131 & 122.7 & 9.51 & A193 & 125.1 & 8.85 & $\mathrm{~S} 246$ & 115.2 & 7.52 \\
\hline Y67 & 115.2 & 8.04 & E132 & 117.7 & 8.56 & S194 & 115.2 & 7.43 & $\mathrm{R} 247$ & 121.5 & 8.10 \\
\hline A70 & 124.1 & 9.18 & E133 & 121.2 & 7.51 & K195 & 119.8 & 6.80 & N248 & 126.1 & 7.29 \\
\hline S71 & 111.6 & 7.65 & K134 & 122.8 & 8.70 & L196 & 116.8 & 8.67 & & & \\
\hline
\end{tabular}

Table 1: Backbone ${ }^{1} \mathrm{H}$ and ${ }^{15} \mathrm{~N}$ resonance assignments of G3P bound TIM at pH 5.7, $298 \mathrm{~K}$, $10 \mathrm{mM} \mathrm{CD}_{3} \mathrm{CO}_{2} \mathrm{Na}$. The assignments were obtained from the assignments of the free protein (BRMB code 7216) by following the chemical2shifts changes upon titration of the ligand. 


\begin{tabular}{|c|c|c|c|c|c|c|c|c|c|c|c|c|}
\hline \multirow{3}{*}{$\begin{array}{l}\text { residue } \\
4\end{array}$} & \multicolumn{12}{|c|}{$R_{e x}\left(\mathrm{~s}^{-1}\right)$} \\
\hline & \multicolumn{3}{|c|}{$\mathrm{T}=298 \mathrm{~K}$} & \multicolumn{3}{|c|}{$\mathrm{T}=293 \mathrm{~K}$} & \multicolumn{3}{|c|}{$\mathrm{T}=288 \mathrm{~K}$} & \multicolumn{3}{|c|}{$\mathrm{T}=283 \mathrm{~K}$} \\
\hline & 1.1 & 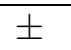 & 0.6 & -0.6 & \pm & 2.5 & 0.5 & \pm & 0.2 & $\overline{1.3}$ & \pm & 3.2 \\
\hline 5 & -3.0 & \pm & 0.5 & -9.9 & \pm & 2.2 & -8.8 & \pm & 0.2 & -5.8 & \pm & 3.3 \\
\hline 10 & -2.2 & \pm & 0.5 & -6.0 & \pm & 2.0 & -5.6 & \pm & 0.2 & -2.7 & \pm & 3.3 \\
\hline 12 & & nd & & 0.6 & \pm & 2.1 & 2.4 & \pm & 0.1 & 3.8 & \pm & 2.9 \\
\hline 13 & -3.8 & \pm & 0.5 & -6.8 & \pm & 1.5 & -5.2 & \pm & 0.2 & -4.8 & \pm & 3.5 \\
\hline 14 & & nd & & 0.4 & \pm & 2.0 & 2.6 & \pm & 0.4 & 3.8 & \pm & 2.9 \\
\hline 15 & 0.2 & \pm & 0.4 & 3.1 & \pm & 0.8 & 3.4 & \pm & 0.8 & -0.3 & \pm & 2.3 \\
\hline 17 & & nd & & -2.9 & \pm & 1.9 & -2.6 & \pm & 0.7 & -3.9 & \pm & 4.1 \\
\hline 18 & & nd & & -0.9 & \pm & 2.6 & -5.4 & \pm & 2.5 & -1.1 & \pm & 4.3 \\
\hline 21 & -0.7 & \pm & 0.5 & -3.3 & \pm & 1.6 & -3.1 & \pm & 0.6 & -3.7 & \pm & 3.8 \\
\hline 23 & 0.0 & \pm & 0.2 & 3.6 & \pm & 2.9 & 2.3 & \pm & 0.9 & -1.0 & \pm & 3.8 \\
\hline 28 & 3.0 & \pm & 0.6 & 0.7 & \pm & 1.9 & 2.8 & \pm & 0.4 & 3.2 & \pm & 3.0 \\
\hline 29 & -0.7 & \pm & 0.4 & -1.5 & \pm & 1.7 & -0.3 & \pm & 0.9 & 0.6 & \pm & 3.1 \\
\hline 30 & -3.2 & \pm & 0.3 & -7.1 & \pm & 1.8 & -7.1 & \pm & 0.9 & -7.4 & \pm & 3.6 \\
\hline 32 & 0.0 & \pm & 0.4 & -1.8 & \pm & 1.8 & -1.1 & \pm & 0.5 & -1.7 & \pm & 2.4 \\
\hline 34 & 0.8 & \pm & 0.3 & -1.2 & \pm & 1.2 & -2.8 & \pm & 0.1 & -0.5 & \pm & 3.0 \\
\hline 35 & 0.0 & \pm & 0.2 & -0.4 & \pm & 1.3 & 0.1 & \pm & 0.2 & -3.6 & \pm & 2.9 \\
\hline 36 & 0.4 & \pm & 0.2 & 3.8 & \pm & 1.9 & 6.0 & \pm & 0.1 & 5.5 & \pm & 2.1 \\
\hline 38 & 0.4 & \pm & 0.3 & -1.3 & \pm & 2.4 & -0.2 & \pm & 0.6 & -0.5 & \pm & 2.7 \\
\hline 45 & 1.4 & \pm & 0.4 & 1.6 & \pm & 1.4 & 1.9 & \pm & 1.8 & -2.3 & \pm & 2.8 \\
\hline 47 & -3.0 & \pm & 0.4 & -6.0 & \pm & 1.5 & -6.9 & \pm & 0.6 & -7.2 & \pm & 3.7 \\
\hline 48 & 0.8 & \pm & 0.8 & -1.4 & \pm & 1.3 & -1.6 & \pm & 0.4 & -2.6 & \pm & 3.4 \\
\hline 49 & 1.3 & \pm & 0.6 & -1.4 & \pm & 1.9 & -0.5 & \pm & 0.2 & -0.3 & \pm & 3.1 \\
\hline 53 & -0.6 & \pm & 0.7 & 4.7 & \pm & 2.6 & 6.6 & \pm & 1.9 & 9.3 & \pm & 3.5 \\
\hline 54 & -0.9 & \pm & 0.6 & -3.2 & \pm & 1.7 & -2.1 & \pm & 0.4 & -2.7 & \pm & 3.5 \\
\hline 55 & -2.5 & \pm & 0.7 & -6.4 & \pm & 2.2 & -5.3 & \pm & 0.4 & -3.9 & \pm & 2.9 \\
\hline 56 & -0.8 & \pm & 0.3 & -0.1 & \pm & 2.3 & 0.5 & \pm & 0.6 & 0.2 & \pm & 2.1 \\
\hline 58 & 1.6 & \pm & 0.2 & 1.0 & \pm & 2.3 & 1.8 & \pm & 0.4 & 3.8 & \pm & 2.6 \\
\hline 64 & & nd & & -1.9 & \pm & 1.6 & -1.4 & \pm & 0.9 & 2.4 & \pm & 3.1 \\
\hline 66 & -0.2 & \pm & 0.2 & 2.1 & \pm & 2.3 & 1.0 & \pm & 0.4 & 0.3 & \pm & 3.1 \\
\hline 67 & 3.2 & \pm & 0.4 & 3.5 & \pm & 1.8 & 4.0 & \pm & 0.3 & 3.5 & \pm & 2.9 \\
\hline 70 & -0.7 & \pm & 0.8 & -1.4 & \pm & 0.7 & -3.9 & \pm & 1.0 & -2.4 & \pm & 2.6 \\
\hline 71 & 0.0 & \pm & 0.3 & 1.0 & \pm & 1.8 & 3.5 & \pm & 0.3 & 1.5 & \pm & 2.8 \\
\hline 72 & -0.9 & \pm & 0.4 & -1.1 & \pm & 1.6 & 1.2 & \pm & 0.9 & -0.8 & \pm & 2.5 \\
\hline 73 & -1.7 & \pm & 0.1 & -3.4 & \pm & 0.7 & -5.2 & \pm & 0.4 & -6.6 & \pm & 2.9 \\
\hline 74 & 1.2 & \pm & 0.1 & 6.3 & \pm & 1.6 & 6.4 & \pm & 0.9 & 4.9 & \pm & 3.0 \\
\hline 76 & -1.8 & \pm & 1.0 & -0.1 & \pm & 3.0 & 1.1 & \pm & 2.9 & 0.5 & \pm & 3.3 \\
\hline
\end{tabular}




\begin{tabular}{|c|c|c|c|c|c|c|c|c|c|c|c|c|}
\hline 77 & -0.8 & \pm & 0.3 & -0.9 & \pm & 2.1 & -0.3 & \pm & 0.3 & -4.9 & \pm & 3.9 \\
\hline 78 & 2.1 & \pm & 1.0 & 1.7 & \pm & 1.3 & 2.6 & \pm & 0.8 & 1.9 & \pm & 2.9 \\
\hline 79 & 0.0 & \pm & 0.4 & -0.7 & \pm & 1.1 & -0.9 & \pm & 0.9 & -3.7 & \pm & 3.0 \\
\hline 80 & & nd & & -6.8 & \pm & 1.6 & -5.6 & \pm & 0.7 & -5.5 & \pm & 3.3 \\
\hline 81 & 0.9 & \pm & 0.5 & 4.0 & \pm & 1.3 & 0.7 & \pm & 1.5 & 1.7 & \pm & 3.1 \\
\hline 82 & -1.0 & \pm & 0.4 & -0.1 & \pm & 2.0 & -0.8 & \pm & 0.5 & 0.5 & \pm & 3.2 \\
\hline 84 & & nd & & 8.4 & \pm & 2.2 & 13.9 & \pm & 0.2 & 20.4 & \pm & 3.1 \\
\hline 85 & 0.1 & \pm & 0.4 & -0.3 & \pm & 1.6 & 1.2 & \pm & 0.4 & 2.2 & \pm & 2.9 \\
\hline 86 & -0.4 & \pm & 0.6 & 4.6 & \pm & 3.2 & 3.1 & \pm & 2.3 & 3.2 & \pm & 3.7 \\
\hline 87 & -1.9 & \pm & 0.2 & -3.4 & \pm & 2.1 & -2.8 & \pm & 0.4 & -4.4 & \pm & 2.8 \\
\hline 94 & -0.4 & \pm & 3.2 & 0.5 & \pm & 1.5 & -1.6 & \pm & 1.2 & -3.6 & \pm & 4.0 \\
\hline 95 & & nd & & 1.4 & \pm & 2.3 & 2.0 & \pm & 1.2 & 4.8 & \pm & 3.8 \\
\hline 100 & -1.2 & \pm & 0.4 & -0.4 & \pm & 2.0 & -0.3 & \pm & 0.7 & -1.3 & \pm & 2.9 \\
\hline 101 & & nd & & -2.0 & \pm & 2.2 & -1.3 & \pm & 0.3 & -0.5 & \pm & 3.3 \\
\hline 102 & 1.6 & \pm & 0.3 & 4.9 & \pm & 1.1 & 6.5 & \pm & 2.2 & 2.5 & \pm & 3.7 \\
\hline 103 & & nd & & -3.3 & \pm & 6.2 & -1.9 & \pm & 0.6 & 4.0 & \pm & 4.8 \\
\hline 104 & 0.9 & \pm & 0.2 & 1.3 & \pm & 1.4 & 1.0 & \pm & 0.2 & 1.7 & \pm & 3.1 \\
\hline 105 & -1.0 & \pm & 0.3 & -2.2 & \pm & 1.9 & -1.8 & \pm & 0.3 & -0.4 & \pm & 3.9 \\
\hline 107 & -0.5 & \pm & 0.5 & -0.7 & \pm & 2.2 & 0.0 & \pm & 0.5 & -0.8 & \pm & 2.9 \\
\hline 110 & -4.0 & \pm & 0.2 & -5.0 & \pm & 2.9 & -4.7 & \pm & 1.5 & -6.1 & \pm & 4.2 \\
\hline 118 & -1.0 & \pm & 0.4 & -7.0 & \pm & 2.1 & -5.6 & \pm & 0.4 & -2.7 & \pm & 2.9 \\
\hline 120 & -1.8 & \pm & 0.2 & -2.3 & \pm & 2.3 & -0.5 & \pm & 0.3 & -4.1 & \pm & 2.8 \\
\hline 121 & & nd & & -0.8 & \pm & 2.0 & 1.1 & \pm & 0.2 & 2.4 & \pm & 3.2 \\
\hline 128 & 3.0 & \pm & 1.7 & -2.0 & \pm & 2.0 & -0.7 & \pm & 0.8 & 6.0 & \pm & 3.1 \\
\hline 129 & -1.8 & \pm & 1.7 & -5.4 & \pm & 2.0 & -7.0 & \pm & 1.1 & -2.9 & \pm & 3.8 \\
\hline 130 & -2.5 & \pm & 2.8 & -2.6 & \pm & 2.2 & -4.9 & \pm & 1.6 & 2.4 & \pm & 3.3 \\
\hline 131 & 0.0 & \pm & 2.8 & -0.2 & \pm & 2.1 & -0.1 & \pm & 2.2 & 0.9 & \pm & 5.1 \\
\hline 134 & -3.1 & \pm & 0.6 & -5.5 & \pm & 1.2 & -5.8 & \pm & 0.8 & -3.7 & \pm & 3.5 \\
\hline 136 & 0.9 & \pm & 0.2 & 1.2 & \pm & 1.9 & 2.4 & \pm & 0.6 & 4.1 & \pm & 3.3 \\
\hline 137 & -4.1 & \pm & 0.3 & -8.8 & \pm & 2.0 & -8.0 & \pm & 0.6 & -6.5 & \pm & 3.2 \\
\hline 139 & -1.2 & \pm & 0.4 & -1.1 & \pm & 1.7 & -0.7 & \pm & 0.9 & -4.5 & \pm & 3.1 \\
\hline 142 & & nd & & -3.2 & \pm & 2.1 & -0.8 & \pm & 0.4 & -2.3 & \pm & 3.2 \\
\hline 143 & 2.1 & \pm & 0.2 & 3.6 & \pm & 2.3 & 4.1 & \pm & 0.7 & 1.6 & \pm & 2.9 \\
\hline 146 & -2.1 & \pm & 0.2 & -5.2 & \pm & 2.2 & -3.3 & \pm & 0.5 & -0.9 & \pm & 3.6 \\
\hline 148 & -0.1 & \pm & 1.3 & -0.1 & \pm & 3.4 & 1.8 & \pm & 0.5 & 3.5 & \pm & 2.9 \\
\hline 149 & & nd & & -1.4 & \pm & 1.2 & -0.6 & \pm & 0.4 & -3.3 & \pm & 3.6 \\
\hline 150 & -1.1 & \pm & 0.9 & 3.0 & \pm & 1.8 & 3.1 & \pm & 0.6 & 5.8 & \pm & 3.4 \\
\hline 151 & -0.1 & \pm & 0.5 & -3.2 & \pm & 1.5 & -0.7 & \pm & 0.4 & 1.5 & \pm & 2.9 \\
\hline
\end{tabular}




\begin{tabular}{|c|c|c|c|c|c|c|c|c|c|c|c|c|}
\hline 156 & & nd & & 0.7 & \pm & 1.5 & 0.2 & \pm & 0.5 & 4.4 & \pm & 3.7 \\
\hline 157 & 4.0 & \pm & 1.1 & 5.1 & \pm & 0.8 & 8.6 & \pm & 1.1 & 5.1 & \pm & 3.0 \\
\hline 158 & -0.8 & \pm & 0.2 & -2.0 & \pm & 1.5 & -2.5 & \pm & 0.5 & -2.0 & \pm & 3.2 \\
\hline 159 & 1.5 & \pm & 0.6 & 0.3 & \pm & 1.5 & 1.8 & \pm & 0.5 & 4.4 & \pm & 2.8 \\
\hline 167 & 9.5 & \pm & 1.7 & 12.0 & \pm & 2.2 & 9.8 & \pm & 4.9 & 9.7 & \pm & 11.4 \\
\hline 169 & 0.2 & \pm & 0.5 & 6.3 & \pm & 2.0 & 7.1 & \pm & 1.2 & 7.0 & \pm & 4.1 \\
\hline 170 & 0.7 & \pm & 1.0 & 5.8 & \pm & 1.6 & 8.9 & \pm & 4.2 & 2.0 & \pm & 3.3 \\
\hline 172 & -1.3 & \pm & 0.2 & -3.3 & \pm & 4.7 & -1.6 & \pm & 6.0 & -7.9 & \pm & 10.2 \\
\hline 173 & -1.3 & \pm & 1.8 & 5.1 & \pm & 6.4 & -2.5 & \pm & 2.3 & 2.4 & \pm & 3.3 \\
\hline 174 & 21.8 & \pm & 0.4 & 32.5 & \pm & 2.4 & 32.3 & \pm & 2.8 & 36.3 & \pm & 6.9 \\
\hline 175 & 2.8 & \pm & 0.3 & 8.3 & \pm & 3.1 & 10.7 & \pm & 7.1 & 24.4 & \pm & 10.0 \\
\hline 177 & 9.2 & \pm & 1.8 & 29.3 & \pm & 5.2 & 16.7 & \pm & 5.0 & 19.4 & \pm & 10.7 \\
\hline 179 & 3.6 & \pm & 0.3 & 4.7 & \pm & 1.4 & 7.1 & \pm & 1.5 & 6.1 & \pm & 3.1 \\
\hline 180 & -0.8 & \pm & 0.3 & -4.8 & \pm & 2.7 & -2.7 & \pm & 0.2 & -1.0 & \pm & 3.1 \\
\hline 182 & & nd & & -0.8 & \pm & 2.6 & 0.3 & \pm & 0.5 & 0.6 & \pm & 3.7 \\
\hline 184 & 3.8 & \pm & 0.1 & 7.5 & \pm & 2.2 & 8.5 & \pm & 1.2 & 12.5 & \pm & 3.2 \\
\hline 185 & -1.6 & \pm & 1.5 & -2.4 & \pm & 2.4 & 0.2 & \pm & 0.6 & 2.0 & \pm & 3.1 \\
\hline 193 & 1.8 & \pm & 0.3 & -1.5 & \pm & 2.2 & -0.3 & \pm & 0.5 & -1.6 & \pm & 3.1 \\
\hline 194 & 0.3 & \pm & 0.4 & 2.1 & \pm & 2.0 & 1.8 & \pm & 0.8 & 0.7 & \pm & 2.8 \\
\hline 195 & 0.5 & \pm & 0.7 & 2.5 & \pm & 1.5 & 2.7 & \pm & 0.2 & 1.3 & \pm & 2.9 \\
\hline 196 & -0.2 & \pm & 0.5 & -1.0 & \pm & 2.1 & -0.8 & \pm & 0.9 & -2.4 & \pm & 2.8 \\
\hline 197 & 0.9 & \pm & 0.3 & 2.8 & \pm & 1.6 & 5.2 & \pm & 0.5 & 2.5 & \pm & 2.7 \\
\hline 201 & -0.1 & \pm & 0.3 & -1.9 & \pm & 2.5 & -1.8 & \pm & 0.5 & 1.4 & \pm & 3.0 \\
\hline 203 & -0.6 & \pm & 0.6 & 0.0 & \pm & 1.6 & -0.9 & \pm & 0.4 & -0.3 & \pm & 3.1 \\
\hline 204 & -2.2 & \pm & 0.2 & -1.2 & \pm & 2.2 & -2.8 & \pm & 0.3 & -3.0 & \pm & 3.3 \\
\hline 205 & 1.0 & \pm & 0.6 & -1.8 & \pm & 2.3 & 2.1 & \pm & 0.4 & 5.3 & \pm & 3.4 \\
\hline 213 & 11.9 & \pm & 2.1 & 17.8 & \pm & 4.1 & 20.6 & \pm & 3.7 & 16.0 & \pm & 6.1 \\
\hline 214 & -1.4 & \pm & 2.6 & -4.9 & \pm & 1.4 & -6.3 & \pm & 0.4 & 1.3 & \pm & 3.1 \\
\hline 217 & 1.8 & \pm & 0.4 & 2.3 & \pm & 2.0 & 2.7 & \pm & 1.2 & 1.5 & \pm & 3.7 \\
\hline 219 & -0.9 & \pm & 0.3 & -2.5 & \pm & 2.2 & -3.4 & \pm & 2.0 & -2.7 & \pm & 3.9 \\
\hline 221 & 9.7 & \pm & 0.5 & 21.8 & \pm & 1.4 & 39.2 & \pm & 1.0 & 46.8 & \pm & 6.2 \\
\hline 224 & 0.6 & \pm & 0.5 & -4.5 & \pm & 2.0 & -2.7 & \pm & 1.0 & -0.8 & \pm & 3.1 \\
\hline 225 & -1.0 & \pm & 3.1 & -0.1 & \pm & 1.4 & -1.8 & \pm & 4.1 & 2.6 & \pm & 4.6 \\
\hline 226 & & nd & & -2.7 & \pm & 1.9 & -2.7 & \pm & 0.7 & -4.7 & \pm & 3.6 \\
\hline 227 & -0.3 & \pm & 0.4 & -1.5 & \pm & 2.4 & -0.8 & \pm & 0.4 & -2.0 & \pm & 3.0 \\
\hline 230 & -2.6 & \pm & 0.5 & -1.7 & \pm & 1.5 & -2.6 & \pm & 2.8 & -4.1 & \pm & 5.4 \\
\hline 231 & 1.0 & \pm & 1.1 & -1.8 & \pm & 1.7 & -4.7 & \pm & 0.3 & -0.5 & \pm & 3.1 \\
\hline 235 & 0.5 & \pm & 2.3 & 5.4 & \pm & 2.3 & 1.7 & \pm & 3.8 & 2.5 & \pm & 4.0 \\
\hline
\end{tabular}




\begin{tabular}{rrrrrrrrrrrrr}
\hline 236 & 0.3 & \pm & 0.5 & 3.0 & \pm & 0.5 & 2.4 & \pm & 2.2 & 2.7 & \pm & 3.2 \\
237 & 0.6 & \pm & 0.4 & 1.4 & \pm & 1.5 & 2.7 & \pm & 0.2 & 3.6 & \pm & 3.2 \\
240 & -0.2 & \pm & 0.4 & 1.4 & \pm & 1.3 & 0.9 & \pm & 1.2 & -3.1 & \pm & 3.9 \\
241 & 1.2 & \pm & 0.5 & -1.1 & \pm & 1.4 & 1.3 & \pm & 0.9 & 1.5 & \pm & 3.0 \\
242 & 0.5 & \pm & 0.5 & 1.3 & \pm & 2.0 & 3.2 & \pm & 1.5 & -0.1 & \pm & 3.3 \\
243 & 0.3 & \pm & 0.3 & -0.9 & \pm & 2.4 & 1.3 & \pm & 0.7 & 1.1 & \pm & 2.9 \\
244 & -0.3 & \pm & 0.9 & 2.4 & \pm & 4.2 & -1.0 & \pm & 0.9 & -2.6 & \pm & 4.1 \\
245 & 2.5 & \pm & 0.6 & 3.4 & \pm & 1.9 & 4.9 & \pm & 0.5 & 2.6 & \pm & 2.9 \\
246 & 0.2 & \pm & 0.5 & 0.1 & \pm & 0.6 & -0.7 & \pm & 1.8 & -1.4 & \pm & 4.0 \\
248 & -1.8 & \pm & 0.1 & -3.4 & \pm & 2.1 & -3.7 & \pm & 0.4 & -6.1 & \pm & 3.1 \\
\hline
\end{tabular}

Table 2: Conformational exchange contribution to transverse relaxation, $R_{e x}$, for G3P bound TIM at $298 \mathrm{~K}, 293 \mathrm{~K}, 288 \mathrm{~K}$, and $283 \mathrm{~K}$ at $B_{0}=18.8 \mathrm{~T}$. For nonexchanging residues, Rex is distributed around zero due to variation in magnitude and orientation of the ${ }^{15} \mathrm{~N}$ CSA tensor. nd indicates that data could not be determined. 